\title{
Exponential Stabilization of an Underactuated Surface Vessel
}

\author{
K. Y. PETTERSEN and O. EGELAND \\ Keywords: Nonlinear control, underactuated vehicles, continuous time-varying \\ feedback
}

The paper shows that a large class of underactuated vehicles cannot be asymptotically stabilized by either continuous or discontinuous state feedback. Furthermore, stabilization of an underactuated surface vessel is considered. Controllability properties of the surface vessels is presented, and a continuous periodic time-varying feedback law is proposed. It is shown that this feedback law exponentially stabilizes the surface vessel to the origin, and this is illustrated by simulations.

\section{Introduction}

Control of underactuated vehicles, i.e. vehicles where the control vector has lower dimension than the configuration vector, is a field of increasing interest. It has been studied by e.g. Byrnes and Isidori (1991) who gave results on stabilizability of a class of underactuated vehicles. Leonard (1995) shows how open loop small-amplitude periodic time-varying forcing can be used to control both underactuated spacecraft and underwater vehicles. Morin, Samson, Pomet and Jiang (1995) present smooth time-varying feedback laws that asymptotically stabilize an underactuated spacecraft. Both M'Closkey and Murray (1993) and Morin and Samson (1995) present important tools for analysis and exponential stabilization of underactuated vehicles.

Byrnes and Isidori (1991) show that underactuated vehicles with zero gravitational and buoyant field cannot be asymptotically stabilized by $C^{1}$ state feedback. We here extend their result by considering underactuated vehicles with a more general gravitational and buoyant field. We show that the vehicles cannot be asymptotically stabilized by either continuous or discontinuous state feedback.

Dynamic positioning of surface vessels is required e.g. in many offshore oil field operations such as drilling, pipe-laying and diving support. Critical to the success of a dynamically positioned surface vessel is its capability for accurate and reliable control, subject to environmental disturbances as well as to configuration related changes, such as a reduced number of available control inputs. This reduction may be the result of an actuator failure or a deliberate decision to limit the number of actuators due to e.g. cost and weight considerations.

In this paper we consider control of a surface vessel without side thruster. The problem considered is to find a control law that asymptotically stabilizes the surface vessel to the origin. As open loop control does not compensate for disturbances and

Received 27 January 1997.

(C) IEEE. This article is reprinted from the Proceedings of the IEEE Conference on Decision and Control, Kobe, Japan, December, 1996 with kind permission from the IEEE.

Department of Engineering Cybernetics, Norwegian University of Science and Technology, N-7034, Trondheim, Norway. Phone: +47 735943 76. Fax: +47 735943 99. Email: KristinY.Pettersen@itk.ntnu.no Olav.Egeland@itk.ntnu.no 


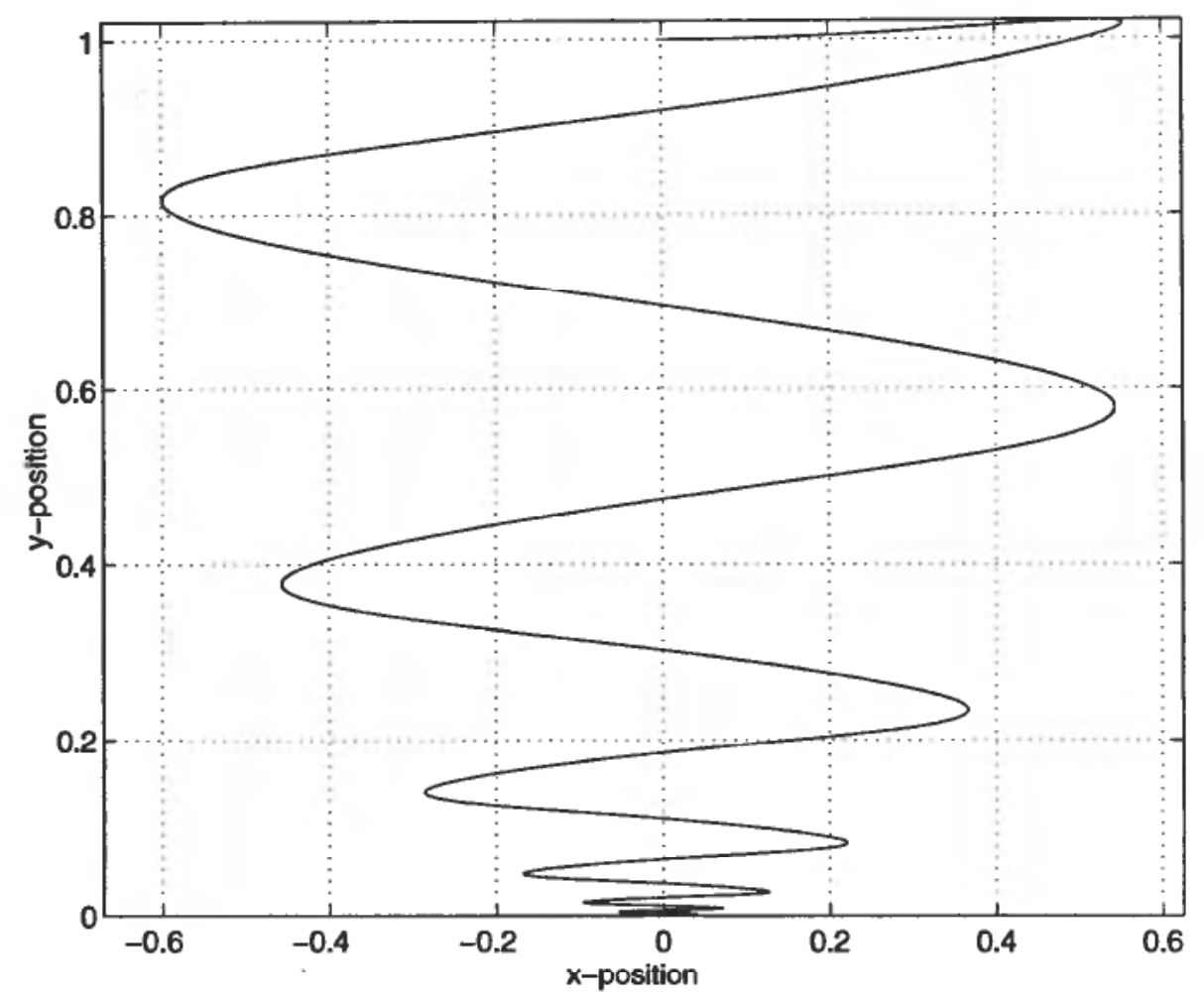

Figure 1. Motion of the surface vessel.

model errors, we seek a feedback law. A question to be answered is whether the surface can be asymptotically stabilized with only two controls.

The stabilizability result gives that the underactuated surface vessel cannot be asymptotically stabilized by either continuous or discontinuous state feedback. We show however that the surface vessel still is locally strongly accessible and small time locally controllable. Furthermore we show that by introducing explicit time-dependence in the controller, the surface vessel can be asymptotically stabilized.

However it is not trivial to find such a feedback law. One approach may be the use of the analysis tools of M'Closkey and Murray (1993) and Morin and Samson (1995). For these tools to apply however, the system has to consist of homogeneous vector fields. The surface vessel model includes trigonometric terms, and is thus not homogeneous with respect to any dilation. To overcome this problem, we propose a global coordinate transformation that renders the system homogeneous.

Furthermore we propose a continuous periodic time-varying feedback law. We show that it asymptotically stabilizes, and in fact yields exponential convergence for the underactuated surface vessel to the origin. This is illustrated by simulations.

The paper is organized as follows. Stabilizability of a class of underactuated vehicles is addressed in Section 2. In Section 3 controllability properties of a surface vessel with only two controls are given. In Section 4 a coordinate transformation is proposed that renders the surface vessel model homogeneous, and a continuous periodic time-varying feedback law is proposed that exponentially stabilizes the surface vessel to the origin. This is illustrated by simulations in Section 5 . 


\section{Stabilizability of underactuated vehicles}

We consider underactuated vehicles of the form

$$
\begin{aligned}
M \dot{v}+C(v) v+D(v) v+g(\eta) & =\left[\begin{array}{l}
\tau \\
0
\end{array}\right] \\
& \dot{\eta}=J(\eta) v
\end{aligned}
$$

where $\eta \in \mathbb{R}^{n_{1}}, v \in \mathbb{R}^{n_{2}}, n_{1} \geq n_{2}, \tau \in \mathbb{R}^{m}, m<n_{2}$. The matrix $M$ is nonsingular and $\dot{M}=0$. The matrix $J$ has full rank $\left(\operatorname{rank}(J)=n_{2}\right)$.

This class includes e.g. underactuated surface vessels, underwater vehicles and spacecraft. The vector $v$ denotes the linear and angular velocities with coordinates in the body-fixed frame, $\eta$ denotes the position and orientation with coordinates in the earth-fixed frame, and $\tau$ denotes the control forces and torques with coordinates in the body-fixed frame. $M$ is the inertia matrix, including added mass. $C(v)$ is the Coriolis and centripetal matrix, also including added mass. $D(v)$ is the damping matrix and $g(\eta)$ is the vector of gravitational and buoyant forces and torques. Equation (2) represents the kinematics.

The following proposition show that the gravitational and buoyant vector, $g(\eta)$, is important for the stabilizability properties of underactuated vehicles. The vector $g(\eta)$ consists of elements corresponding to the actuated dynamics, $g^{a}(\eta)$, and elements corresponding to the unactuated dynamics, $g^{u}(\eta)$ :

$$
\begin{gathered}
g(\eta)=\left[\begin{array}{l}
g^{a}(\eta) \\
g^{u}(\eta)
\end{array}\right] \\
g^{a}(\eta): \mathbb{R}^{n_{1}} \rightarrow \mathbb{R}^{m} g^{u}(\eta): \mathbb{R}^{n_{1}} \rightarrow \mathbb{R}^{n_{2}-m}
\end{gathered}
$$

Proposition 1 Consider the system $(1)-(2)$, and suppose that $(\eta, v)=(0,0)$ is an equilibrium of the system. If $g^{u}(\eta)$ has a zero element then there exists no continuous or discontinuous state feedback law, $\alpha(\eta, v): \mathbb{R}^{n_{1}} \times \mathbb{R}^{n_{2}} \rightarrow \mathbb{R}^{m}$, that makes $(0,0)$ asymptotically stable.

Proof. Consider the mapping $f(\eta, v, \tau): \mathbb{R}^{n_{1}} \times \mathbb{R}^{n_{2}} \times \mathbb{R}^{m} \rightarrow \mathbb{R}^{n_{1}+n_{2}}$ defined by

$$
f(\eta, v, \tau)=\left[\begin{array}{c}
J(\eta) v \\
-M^{-1}\left(C(v) v+D(v) v+g(\eta)-\left[\begin{array}{l}
\tau \\
0
\end{array}\right]\right)
\end{array}\right]
$$

Without loss of generality, we assume that

$$
g^{u}(\eta)=\left[\begin{array}{c}
g_{1}^{u}(\eta) \\
0
\end{array}\right] g_{1}^{u}(\eta): \mathbb{R}^{n_{1}} \rightarrow \mathbb{R}^{n_{2}-m-1}
$$

Consider points of the form

$$
\varepsilon=\left[\begin{array}{c}
0_{n_{1} \times 1} \\
M^{-1}\left[\begin{array}{c}
\alpha^{1} \\
\alpha^{2} \\
\beta
\end{array}\right]
\end{array}\right]
$$

where $\alpha^{1} \in \mathbb{R}^{m}, \alpha^{2} \in \mathbb{R}^{n_{2}-m-1}$ are arbitrary vectors, and $\beta \in \mathbb{R}$ is an arbitrary non-zero number. As $J(\eta)$ has full rank, the equation $f(\eta, v, \tau)=\varepsilon$ implies $v=0$. Thus it implies 


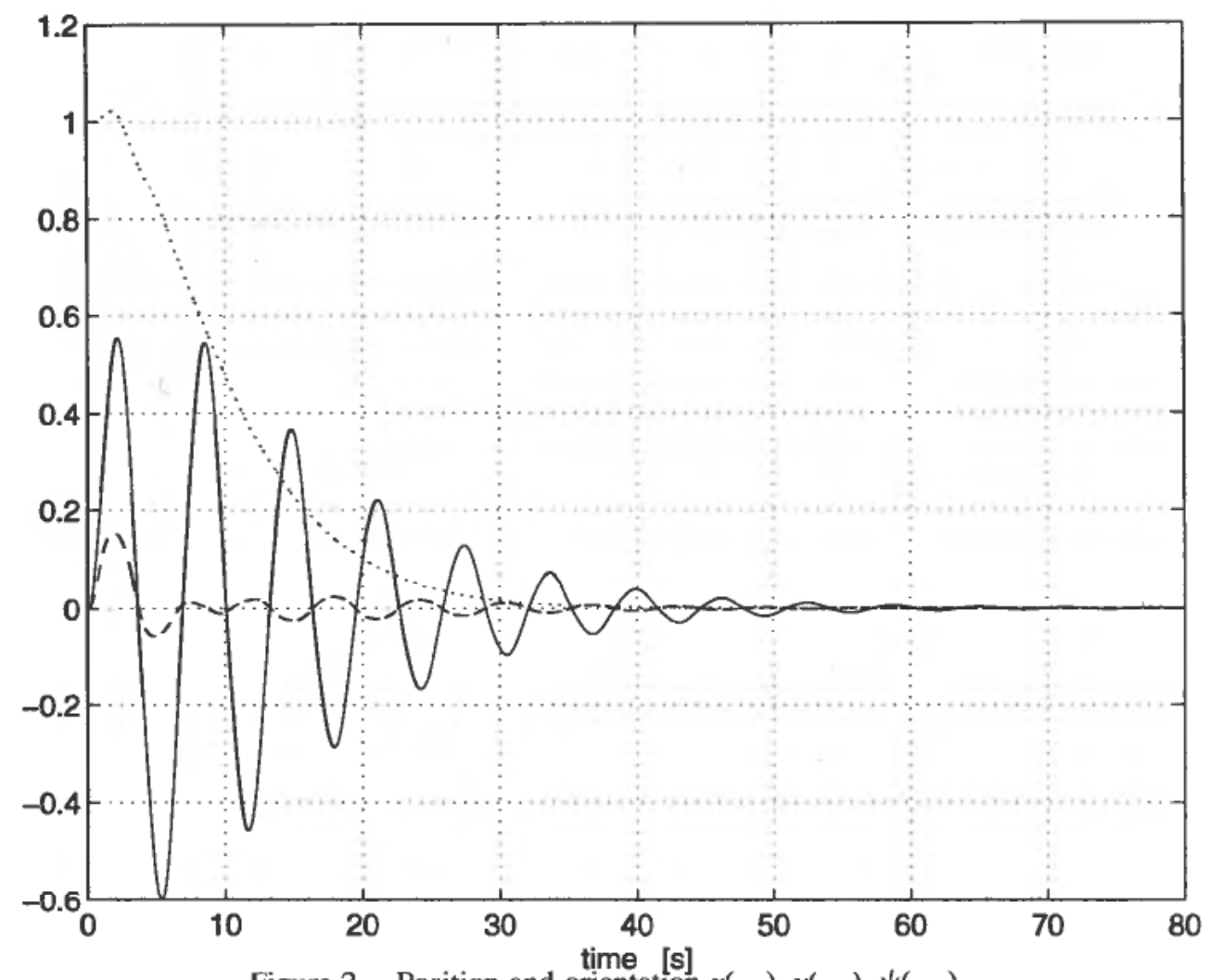

Figure 2. Position and orientation $x(-), y(\cdots), \psi(--)$.

$$
\left[\begin{array}{c}
\tau-g^{a}(\eta) \\
-g_{1}^{u}(\eta) \\
0
\end{array}\right]=\left[\begin{array}{c}
\alpha^{1} \\
\alpha^{2} \\
\beta
\end{array}\right]
$$

which has no solution $(\eta, v, \tau)$ as $\beta$ is non-zero. As $M$ is constant and nonsingular, and $\alpha^{1}, \alpha^{2}, \beta$ are arbitrary vectors, points of the form $\varepsilon$ are contained in every neighborhood of zero in $\mathbb{R}^{n_{1}+n_{2}}$. Thus the mapping $f$ is not onto any neighborhood of 0 in $\mathbb{R}^{n_{1}+n_{2}}$. This implies that there does not exist any continuous state feedback $\alpha(\eta, v): \mathbb{R}^{n_{1}} \times \mathbb{R}^{m_{2}} \rightarrow \mathbb{R}^{m}$ such that $(\eta, v)=(0,0)$ is an asymptotically stable equilibrium (Zabczyk 1989). As the system (1)-(2) is affine and cannot be asymptotically stabilized by continuous state feedback, it cannot be asymptotically stabilized by discontinuous state feedback either (Coron and Rosier 1994).

\section{Controllability properties of an underactuated surface vessel}

We consider the surface vessel described by the following model:

$$
\begin{aligned}
M \dot{v}+C(v) v+D v & =\tau \\
\dot{\eta} & =J(\eta) \gamma
\end{aligned}
$$

where

$$
C(v)=\left[\begin{array}{ccc}
0 & 0 & -m_{22} v \\
0 & 0 & m_{11} u \\
m_{22} v & -m_{11} u & 0
\end{array}\right]
$$




$$
J(\eta)=\left[\begin{array}{ccc}
\cos \psi & -\sin \psi & 0 \\
\sin \psi & \cos \psi & 0 \\
0 & 0 & 1
\end{array}\right]
$$

$M=\operatorname{diag}\left\{m_{11}, m_{22}, m_{33}\right\}, D=\operatorname{diag}\left\{d_{11}, d_{22}, d_{33}\right\}$.

The vector $v=[u, v, r]^{T}$ denotes linear velocities in surge and sway, and angular velocity in yaw. The vector $\eta=[x, y, \psi]^{T}$ denotes position and orientation in earth-fixed coordinates. The vector $\tau=\left[\tau_{1}, \tau_{2}, \tau_{3}\right]^{T}$ denotes the control forces in surge and sway, and control torque in yaw. The inertia matrix $M$ and the damping matrix $D$ are constant and positive definite.

We consider the case where the surface vessel has no side thruster, i.e. $\tau_{2}=0$, because this is a common thruster configuration. However, the controllability analysis and control synthesis of this paper is easily extended to the cases where $\tau_{1}$ or $\tau_{3}$ are missing.

From Proposition 1 we know that the surface vessel cannot be asymptotically stabilized by either continuous or discontinuous state feedback, if any of the controls are missing. However we show here that the surface vessel yet is locally strongly accessible and small time locally controllable. Furthermore we show that by introducing explicit time dependence in the controller, asymptotic stability may be obtained.

Let

$$
\begin{gathered}
f(\eta, v)=\left[\begin{array}{c}
J(\eta) v \\
-M^{-1}(C(v)+D) v
\end{array}\right] \\
g_{1}=\left[\begin{array}{llllllllll}
0 & 0 & 0 & \frac{1}{m_{11}} & 0 & 0
\end{array}\right]^{T} \quad g_{2}=\left[\begin{array}{llllll}
0 & 0 & 0 & 0 & 0 & \frac{1}{m_{33}}
\end{array}{ }^{T}\right.
\end{gathered}
$$

Then the system (8)-(9) with $\tau_{2}=0$ may be written

$$
\left[\begin{array}{l}
\dot{\eta} \\
\dot{v}
\end{array}\right]=f(\eta, v)+g_{1} \tau_{1}+g_{2} \tau_{3}
$$

Lemma 1 The underactuated surface vessel (14) is locally strongly accessible $\forall(\eta, v) \in \mathbb{R}^{6}$.

Proof. The vector fields $g_{1}, g_{2},\left[f, g_{1}\right],\left[f, g_{2}\right],\left[g_{2},\left[f, g_{1}\right]\right],\left[g_{2},\left[\left[g_{1}, f\right], f\right]\right]$ span a sixdimensional space at every $(\eta, v) \in \mathbb{R}^{6}$. Thus the strong accessibility rank condition is satisfied, and consequently (14) is locally strongly accessible $\forall(\eta, v) \in \mathbb{R}^{6}$ (Nijmeijer and van der Schaft 1990).

Proposition 2 The underactuated surface vessel (14) is small time locally controllable from any equilibrium.

Proof. From Lemma 1 we know that the system (14) is locally strongly accessible from every $(\eta, v) \in \mathbb{R}^{6}$, and as the vector fields $f, g_{1}, g_{2}$ are real-analytic, this implies that the Lie algebra rank condition is satisfied $\forall(\eta, v) \in \mathbb{R}^{6}$ (Sussmann 1987). We note from the proof of Lemma 1 that any bracket with degree greater than 4 can be expressed as a linear combination of lower order brackets. We also note that the degree of 'bad' brackets must be odd (Sussmann 1987). The 'bad' bracket of degree 1 is $f$, which vanishes at any equilibrium. The 'bad' brackets of degree 3 are $\left[g_{1},\left[f, g_{1}\right]\right]$ and $\left[g_{2},\left[f, g_{2}\right]\right]$ which are identically zero vector fields. Consequently, the system (14) 
satisfies the Sussmann Theorem, and is thus small time locally controllable from any equilibrium (Sussmann 1987).

The following proposition shows that a time-varying feedback law that is non-smooth at the origin, can asymptotically stabilize the surface vessel. An almost smooth periodic time-varying feedback law for the system (14) is a function $\tau$ in $C^{0}\left(\mathbb{R}^{6} \times \mathbb{R} ; \mathbb{R}^{2}\right)$ of class $C^{\infty}$ on $\left(\mathbb{R}^{6}\{0\}\right) \times \mathbb{R}$ that satisfies

$$
\begin{aligned}
\tau(0, t) & =0 \quad \forall \mathrm{t} \in \mathbb{R} \\
\tau(\eta, v, t+T) & =\tau(\eta, v, t) \quad \forall t \in \mathbb{R}
\end{aligned}
$$

Proposition 3 The underactuated surface vessel (14) is locally asymptotically stabilizable in small time by means of an almost smooth periodic time-varying feedback law.

Proof. By (Coron 1995), as the dimension of the vector $(\eta, v)$ is more than 4 and the vector fields $f, g_{1}, g_{2}$ are analytic, this follows from Proposition 2 .

\section{Exponential stabilization of the underactuated surface vessel}

From Proposition 3 we know that the surface vessel can be asymptotically stabilized by an almost smooth periodic time-varying feedback law. However it is not trivial to find such a feedback law. M'Closkey and Murray (1993) and Morin and Samson (1995) have developed important analysis tools for continuous systems when the vector fields are homogeneous with respect to a given dilation. The drift vector field of the surface vessel (14) however includes trigonometric terms, and is thus not homogeneous with respect to any dilation.

However, the homogeneity property is coordinate dependent. Motivated by this we seek a coordinate transformation that renders the system homogeneous. We propose the following coordinate transformation, which is a global diffeomorphism:

$$
\begin{aligned}
& z_{1}=\cos (\psi) x+\sin (\psi) y \\
& z_{2}=-\sin (\psi) x+\cos (\psi) y \\
& z_{3}=\psi
\end{aligned}
$$

The state equations of the surface vessel are then:

$$
\begin{aligned}
\dot{z}_{1} & =u+z_{2} r \\
\dot{z}_{2} & =v-z_{1} r \\
\dot{z}_{3} & =r \\
\dot{u} & =\frac{m_{22}}{m_{11}} v r-\frac{d_{11}}{m_{11}} u+\frac{1}{m_{11}} \tau_{1} \\
\dot{v} & =-\frac{m_{11}}{m_{33}} u r \frac{m_{11}}{m_{22}} u r-\frac{d_{22}}{m_{22}} v \\
\dot{r} & =\frac{m_{11}-m_{22}}{m_{33}} u v-\frac{d_{33}}{m_{33}} r+\frac{1}{m_{33}} \tau_{3}
\end{aligned}
$$

Proposition 4 Consider the functions

$$
\begin{aligned}
& u_{d}(z, v, t)=-k_{1} z_{1}+\rho^{\alpha}(z, v) \sin (t / \varepsilon) \\
& r_{d}(z, v, t)=-k_{2} z_{3}+\frac{2}{\rho^{\alpha}(z, v)}\left(k z_{2}+d v\right) \sin (t / \varepsilon)
\end{aligned}
$$




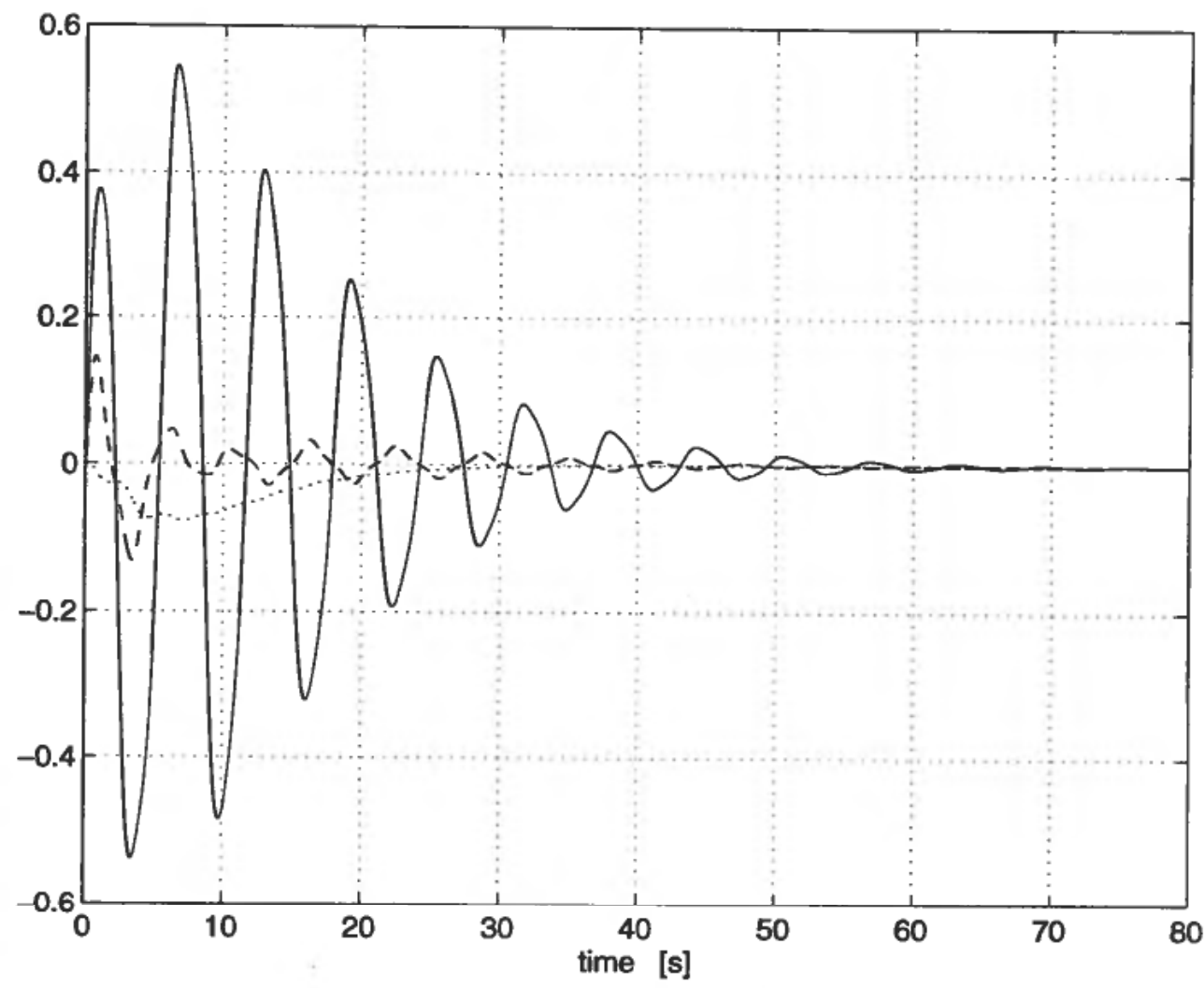

Figure 3. Velocities $u(-), v(\cdots), r(--)$.

where $k_{1}, k_{2}>0, k>0, d>0$ and $\rho^{\alpha}(z, v)$ is any homogeneous norm associated with the dilation $\delta_{\lambda}^{a}(z, v, t)=\left(\lambda z_{1}, \lambda^{2} z_{2}, \lambda z_{3}, \lambda^{2} v, t\right)$. Given the following continuous timevarying feedback law

$$
\begin{gathered}
\tau_{1}(z, v, t)=-\left(k_{3} m_{11}-d_{11}\right) u+k_{3} m_{11} u_{d}(z, v, t) \\
\tau_{3}(z, v, t)=-\left(k_{4} m_{33}-d_{33}\right) r+k_{4} m_{33} r_{d}(z, v, t)
\end{gathered}
$$

Then there exists an $\varepsilon_{0}>0$ such that for any $\varepsilon \in\left(0, \varepsilon_{0}\right)$ and for positive and large enough parameters $k_{3}, k_{4}$, the feedback law (20) locally exponentially stabilizes the origin of the system (14).

Proof. The system (18)-(20) may be written

$$
\left[\begin{array}{l}
\dot{z} \\
\dot{v}
\end{array}\right]=l(z, v, t)+h(z, v, t)
$$

where

$$
l(z, v, t)=\left[\begin{array}{c}
u \\
v-z_{1} r \\
r \\
\frac{1}{m_{11}}\left(\tau_{1}-d_{11} u\right) \\
-\frac{m_{11}}{m_{22}} u r-\frac{d_{22}}{m_{22}} v \\
\frac{1}{m_{33}}\left(\tau_{3}-d_{33} r\right)
\end{array}\right]
$$




$$
h(z, v, t)=\left[\begin{array}{c}
z_{2} r \\
0 \\
0 \\
\frac{m_{22}}{m_{11}} v r \\
0 \\
\frac{m_{11}-m_{22}}{m_{33}} u v
\end{array}\right]
$$

Consider the dilation

$$
\delta_{\lambda}^{\beta}(z, v, t)=\left(\lambda z_{1}, \lambda^{2} z_{2}, \lambda z_{3}, \lambda u, \lambda^{2} v, \lambda r, t\right)
$$

As the functions $\tau_{1}$ and $\tau_{3}$ are homogeneous of degree 1 with respect to the dilation, and continuous for $(z, v) \neq 0$, they are also continuous at zero. The vector function $l$ is thus continuous. It is furthermore time-periodic and $l(0,0, t)=0$. The vector function $h$ is continuous. The system

$$
\left[\begin{array}{c}
\dot{z} \\
\dot{v}
\end{array}\right]=l(z, v, t)
$$

is homogeneous of degree 0 with respect to the dilation $\delta_{\lambda}^{B}$, and the vector field $h$ is homogeneous of degree 2 with respect to $\delta$. Thus the solution $(z, v)=(0,0)$ of the system (21) is locally $\rho^{\beta}$-exponentially stable if the equilibrium $(z, v)=(0,0)$ is a locally asymptotically stable equilibrium of the system (25) (Morin and Samson, Prop. 2).

We reduce the system (25), by defining $u_{d} \stackrel{\text { def }}{=} u$ and $r_{d} \stackrel{\text { def }}{=} r$ as control variables.

$$
\left[\begin{array}{c}
\dot{z} \\
\dot{v}
\end{array}\right]=\left[\begin{array}{c}
u_{d} \\
v-z_{1} r_{d} \\
r_{d} \\
-\frac{m_{11}}{m_{22}} u_{d} r_{d}-\frac{\mathrm{d}_{22}}{m_{22}} v
\end{array}\right]
$$

With controls given by (19) the averaged system of (26) is

$$
\left[\begin{array}{c}
\dot{z} \\
\dot{v}
\end{array}\right]=\left[\begin{array}{c}
-k_{1} z_{1} \\
v+k_{2} z_{1} z_{3} \\
-k_{2} z_{3} \\
-\frac{m_{11}}{m_{22}} k_{1} k_{2} z_{1} z_{3}-\frac{m_{11}}{m_{22}}\left(k z_{2}+d v\right)-\frac{d_{22}}{m_{22}} v
\end{array}\right]
$$

The linearization of $(27)$ about $(z, v)=(0,0)$ is obviously locally asymptotically stable. Furthermore the system (26) is continuous, time-periodic and homogeneous of degree 0 with respect to the dilation $\delta_{\lambda}^{\alpha}$. Thus there exists an $\varepsilon_{0}>0$ such that for any $\varepsilon \in\left(0, \varepsilon_{0}\right)$ the origin of (26) is locally exponentially stable (M'Closkey and Murray 1993, Th. 4.1).

The functions $u_{d}(z, v, t)$ and $r_{d}(z, v, t)$ are continuous, time-periodic, differentiable with respect to $t$, to class $C^{1}$ on $\left(\mathbb{R}^{3} \times \mathbb{R}-\{0,0\}\right) \times \mathbb{R}$, and homogeneous of degree 1 with respect to the dilation $\delta_{\lambda}^{\alpha}$. The equations for $u$ and $r$ in (25) with control (20) are

$$
\begin{gathered}
\dot{u}=-k_{3}\left(u-u_{d}(z, v, t)\right) \\
\dot{r}=-k_{4}\left(r-r_{d}(z, v, t)\right)
\end{gathered}
$$

Thus for positive and large enough values of $k_{3}, k_{4}$ the origin $(z, v)=(0,0)$ of the system 


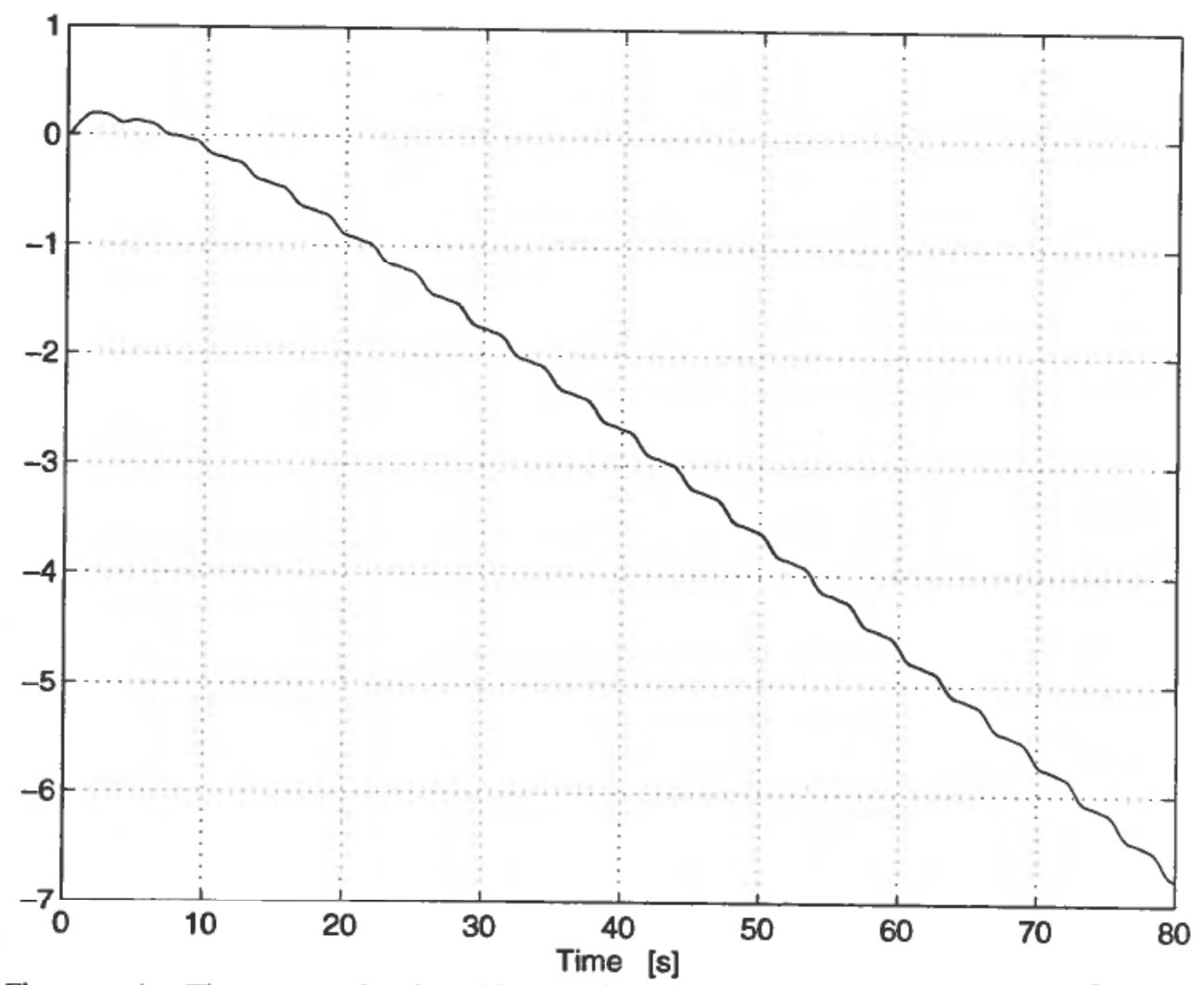

Figure 4. The natural logarithm of the homogeneous norm $\rho^{\beta}(z, v)=$

$$
\sqrt{z_{1}^{2}+\left|z_{2}\right|+z_{3}^{2}+u^{2}+|v|+r^{2}} \text {. }
$$

(25) with control (19)-(20) is locally asymptotically stable (Morin and Samson 1995, Cor. 1).

Thus $(z, v)=(0,0)$ is a locally exponentially stable equilibrium of (21) (Morin and Samson 1995, Prop. 2). As (17) is a diffeomorphism, $(\eta, v)=(0,0)$ is therefore a locally exponentially stable equilibrium of (14)-(20).

Remark 1 The controller in Proposition 4 also exponentially stabilizes surface vessels with higher order damping, i.e.

$$
D(v)=\operatorname{diag}\left\{d_{11}+\sum_{i \geq 2} d_{u^{i}}|u|^{i-1}, d_{22}+\sum_{i \geq 2} d_{v^{i}}|v|^{i-1}, d_{33}+\sum_{i \geq 2} d_{r^{i}}|r|^{i-1}\right\}
$$

This is easily shown by including the higher order terms into $h(z, v, t)$.

\section{Simulations}

The action of the control law (19)-(20) has been simulated with control parameters

$$
\begin{gathered}
k_{1}=1.5, k_{2}=1.5, k_{3}=10, k_{4}=10 \\
k=0.2, \mathrm{~d}=1.7, \varepsilon=1
\end{gathered}
$$


The homogeneous norm used in the control law was

$$
\rho^{\alpha}(z, v)=\sqrt{z_{1}^{2}+\left|z_{2}\right|+z_{3}^{2}+|v|}
$$

and the initial conditions were:

$$
[x(0), y(0), \psi(0), u(0), v(0), r(0)]^{T}=[0,1,0,0,0,0]^{T}
$$

The feedback law (19)-(20) make the origin of the underactuated surface vessel (14) exponentially stable for small enough values of $\varepsilon$ and large enough values of $k_{3}$ and $k_{4}$. The simulations illustrate that $\varepsilon$ does not have to be very small nor $k_{3}$ or $k_{4}$ very large.

We see that the natural logarithm of the homogenous norm $\rho^{\beta}(z, v)$ is upper bounded by a decreasing straight line. This illustrates the exponential convergence of the system to zero.

We note from the analysis that the only model parameters that the performance of the controller may depend on exact knowledge of, are $d_{11}$ and $d_{33}$. We have simulated the action of the controller when the terms $d_{11} u$ and $d_{33} r$ in (20) are neglected, and also when $d_{11}$ and $d_{33}$ have much larger values than the known model parameters, and the controller still provides exponential stability. This indicates that the controller is robust against model parameter uncertainty.

\section{Acknowledgments}

The authors would like to thank O.J. Sørdalen for many interesting discussions. The research was partly supported by The Research Council of Norway.

\section{REFERENCES}

BYRNES, C. and IsIDORI, A. (1991). On the attitude stabilization of rigid space-craft. Automatica, 27, 87-95.

CORON, J.-M. (1995). On the stabilization in finite time of locally controllable systems by means of continuous time-varying feedback law. SIAM J. of Control and Optimization, 33(3), 804-833.

Coron, J.-M. and Rosier, L. (1994). A relation between continuous time-varying and discontinuous feedback stabilization. J. of Math. Syst. Estimation, and Control, 4(1), $67-84$.

LEONARD, N. E. (1995). Periodic forcing, dynamics and control of underactuated spacecraft and underwater vehicles. Proc. 34th IEEE Conf. on Decision and Control, New Orleans, LA, pp. 3980-3985.

M'ClOSKEY, R. T. and MurRAY, R. M. (1993). Nonholonomic systems and exponential convergence: some analysis tools. Proc. 32nd IEEE Conf. on Decision and Control, San Antonio, Texas, pp. 943-948.

MORIN, P. and SAMSON, C. (1995). Time-varying exponential stabilization of the attitude of a rigid spacecraft with two controls. Proc. 34th IEEE Conf. on Decision and Control, New Orleans, LA, pp. 3988-3993.

Morin, P., SAMSON, C., POMET, J.-B. and JiANG, Z.-P. (1995). Time-varying feedback stabilization of the attitude of a rigid spacecraft with two controls. Syst. and Control Letters, 25(5), 375-385.

Nijmejer, H. and VAN DeR SChaft, A. (1990). Nonlinear Dynamical Control Systems, Springer-Verlag, New York.

SUSSMANN, H. (1987). A general theorem on local controllability. SIAM J. of Control and Optimization, 25(1), 158-194.

ZABCZYK, J. (1989). Some comments on stabilizability. Appl. Math. and Optimization, 19, 1-9. 